\title{
SOME ERROR ESTIMATES FOR THE NUMERICAL APPROXIMATION OF SURFACE INTEGRALS
}

\author{
KURT GEORG AND JOHANNES TAUSCH
}

\begin{abstract}
Recently, the first author introduced a new approach to the numerical quadrature of surface integrals in the context of boundary element methods. It is assumed that a global parametrization $m$ of the surface is only indirectly given (e.g., via an iterative method) and that $m$ is not accessible analytically. Of particular interest are parametrizations which are based on automatic triangulations of surfaces. In order to avoid an explicit reference to the partial derivatives of $m$, modified trapezoidal and midpoint rules were introduced. The present paper discusses some error estimates for these methods. The estimates are surprisingly difficult since $\mathscr{O}\left(h^{3}\right)$-terms have to be shown to cancel; this does not occur in the expansion of the standard rules.
\end{abstract}

\section{INTRODUCTION}

Efficient numerical approximations of surface integrals are important in boundary element methods; see, e.g., Atkinson [1, 2], Georg and Widmann [4], Hackbusch [5].

Recently, Georg [3] introduced a new approach to the numerical quadrature of surface integrals. It was assumed that the surface $\mathscr{B} \subset \mathbb{R}^{3}$ was modeled via a piecewise linear approximation (triangulation). Such approximations are typically used in panel methods. Hence, a parametrization $m$ of the surface $\mathscr{B}$ is only indirectly given, e.g., via an iterative method. The main purpose of the new method is to avoid the handling of the partial derivatives of $m$ via finite differences or interpolation.

Throughout this paper, let $\mathscr{S}$ denote a smooth piece of the surface $\mathscr{B}$ and $m: \sigma \rightarrow \mathscr{S}$ a smooth (parameter) map. For the purposes of our discussion it is enough to consider the case that $\sigma=\{(s, t): 0 \leq s, t, s+t \leq 1\}$ is a standard triangle which is isomorphically mapped onto $\mathscr{S}$. We consider the task of numerically approximating

$$
\int_{\mathscr{S}} f d S
$$

Received by the editor November 5, 1992 and, in revised form, April 22, 1993.

1991 Mathematics Subject Classification. Primary 65D30, 65N38.

Key words and phrases. Numerical surface integration, error estimates of quadrature rules, boundary element method, piecewise linear surface approximation, extrapolation method, quadrature formula, trapezoidal rule, midpoint rule .

Partially supported by the National Science Foundation under grant number DMS-9104058. 
Here, $d S$ indicates the usual measure on $\mathscr{S}$ (surface element).

The standard approach to this task is to consider the equivalent integral in planar coordinates

$$
\int_{\mathscr{S}} f d S=\int_{\sigma} f(m(s, t))\left\|m_{s} \times m_{t}\right\| d s d t .
$$

Here, $m_{s}$ denotes the partial derivative of $m$ with respect to the parameter $s$. In order to numerically approximate (2), a standard approach is to subdivide $\sigma$ into small triangles $\sigma_{i}$; this corresponds to a subdivision of the surface $\mathscr{S}$ into the pieces $\mathscr{S}_{i}:=m\left(\sigma_{i}\right)$. Then an approximation of the integral can be obtained via one of the composite rules

$$
\begin{aligned}
& \int_{\mathscr{S}} f d S=\sum_{i} \int_{\sigma_{i}}(f \circ m) \gamma d s d t \\
& \approx \sum_{i} \frac{1}{3} \sum_{j=0}^{2} f\left(m\left(x_{i, j}\right)\right) \gamma\left(x_{i, j}\right) \operatorname{area}\left(\sigma_{i}\right), \\
& \int_{\mathscr{S}} f d S=\sum_{i} \int_{\sigma_{i}}(f \circ m) \gamma d s d t \\
& \approx \sum_{i} f\left(m\left(x_{i, b}\right)\right) \gamma\left(x_{i, b}\right) \operatorname{area}\left(\sigma_{i}\right) .
\end{aligned}
$$

Here, the vertices and the barycenter of $\sigma_{i}$ are denoted by $x_{i, j}, j=0,1,2$, and $x_{i, b}$, respectively, and $\gamma:=\left\|m_{s} \times m_{t}\right\|$.

Methods (3) and (4) are the respective extensions of the trapezoidal and the midpoint rule for a triangle.

The local error, i.e., the error of each summand, is $\mathscr{O}\left(h^{4}\right)$, where $h=$ $\max _{i} \operatorname{diam}\left(\sigma_{i}\right)$. Under the summation of the composite rule, these local errors lead to a global error $\mathscr{O}\left(h^{2}\right)$. It is also known (in the case of an equidistant subdivision) that the global error can be expanded in terms of $h^{2}$, see Lyness [6].

If the partial derivatives of $m$ are not available analytically, a numerical approximation (e.g., via finite differences or interpolation) may be considered; see, e.g., Atkinson [2]. An alternative approach to approximate (1) that does not use the partial derivatives of $m$ was proposed by Georg [3]:

$$
\begin{aligned}
\int_{\mathscr{S}} f d S & \approx \sum_{i} \frac{1}{3} \sum_{j=0}^{2} f\left(v_{i, j}\right) \text { area }\left[v_{i, 0}, v_{i, 1}, v_{i, 2}\right], \\
\int_{\mathscr{S}} f d S & \approx \sum_{i} f\left(v_{i, b}\right) \operatorname{area}\left[v_{i, 0}, v_{i, 1}, v_{i, 2}\right] .
\end{aligned}
$$

Here, $v_{i, j}$ are the images of the vertices $x_{i, j}$ of $\sigma_{i}$ under $m$ and $\left[v_{i, 0}, v_{i, 1}\right.$, $v_{i, 2}$ ] denotes the triangle spanned by $v_{i, 0}, v_{i, 1}, v_{i, 2}$. The point $v_{i, b}$ denotes the barycenter of $\left[v_{i, 0}, v_{i, 1}, v_{i, 2}\right]$. Therefore, we need to assume that the integrand $f$ is extended to a smooth function defined on some open neighborhood of $\mathscr{S}$ so that for sufficiently small $h$ the value $f\left(v_{i, b}\right)$ is defined.

The main result of the present paper is contained in the following 
Theorem 1. The global discretization error of both composite rules (5) and (6) is $\mathscr{O}\left(h^{2}\right)$.

Hence, our modified rules have the same global order as the corresponding standard composite rules (3) and (4). In contrast to standard rules, however, it is impossible to prove that the local error is of order four in our modified rules. This is the main difficulty we encounter in our analysis. We will show that the $\mathscr{O}\left(h^{3}\right)$-terms cancel under the summation of the composite rule.

In fact, numerical experiments also suggest that the error of the modified methods (5) and (6) have an $h^{2}$-expansion; an analytical demonstration of this observation seems to be difficult, since Lyness's [6] proof for the standard case, which is based on the Euler-Maclaurin sum formula, does not easily carry over to our case.

\section{Proof of the estimate}

In this section we will sketch a proof of the above theorem. The argument is somewhat technical, and therefore we will leave some of the more obvious details to the reader.

The subdivision $\left\{\sigma_{i}\right\}$ of $\sigma$ induces a subdivision $\left\{\mathscr{S}_{i}\right\}$ of $\mathscr{S}$ into curved triangular pieces. The pieces $\mathscr{S}_{i}$ are approximated by the triangles $T_{i}:=$ $\left[v_{0, i}, v_{1, i}, v_{2, i}\right]$ and the surface area of $\mathscr{S}$ is approximated by the area of the triangulation $\mathscr{T}=\left\{T_{i}\right\}$ of $\mathscr{S}$. We assume that the subdivision $\left\{\sigma_{i}\right\}$ of $\sigma$ does not produce arbitrarily small angles. More precisely, there are constants $\alpha_{0}$ and $\alpha_{1}$ independent of $h$ such that

$$
0<\alpha_{0} \leq \alpha \leq \alpha_{1}<\pi
$$

holds for any interior angle $\alpha$ of each $T_{i}$. Thus, the sum in the composite rules (5), (6) is taken over $\mathscr{O}\left(h^{-2}\right)$ terms. Let $P_{i}$ denote the plane in which $T_{i}$ is embedded and $n_{i}$ its normal vector. We project $\mathscr{S}_{i}$ in the direction of $n_{i}$ onto a set $N_{i} \subset P_{i}$ and assume that $h$ is chosen to be sufficiently small such that this projection is one to one. It is convenient to introduce a new parametrization of $\mathscr{S}_{i}$ :

Let $g_{i}(x)$ denote the distance of a point $x \in R^{3}$ from $\mathscr{S}_{i}$ in direction of the normal $n_{i}$ of $P_{i}$, and let $m^{L}: R^{2} \rightarrow P_{i}$ denote the parametrization of the plane $P_{i}$ defined by

$$
m^{L}(s, t)=(1-s-t) v_{0, i}+s v_{1, i}+t v_{2, i}, \quad(s, t) \in \mathbb{R}^{2} .
$$

Furthermore, we parametrize $\mathscr{S}_{i}$ via $m^{i}: N_{i} \rightarrow \mathscr{S}_{i}$ by

$$
m^{i}(v)=v+g_{i}(v) n_{i} .
$$

We will need both parametrizations to prove the first result:

$$
\int_{\mathscr{S}_{i}} f d S=\int_{N_{i}} f \circ m^{i}\left(1+\mathscr{O}\left(h^{2}\right)\right) d P_{i} .
$$

Here, $d P_{i}$ denotes the standard measure in the plane $P_{i}$. 
Proof. For an infinitesimal surface element, we have

(10)

$$
\begin{aligned}
d S & =\left\|\left(m^{i} \circ m^{L}\right)_{s} \times\left(m^{i} \circ m^{L}\right)_{t}\right\| d s d t \\
& =\left\|\left(m^{L}+g_{i}\left(m^{L}\right) n_{i}\right)_{s} \times\left(m^{L}+g_{i}\left(m^{L}\right) n_{i}\right)_{t}\right\| d s d t \\
& =\left\|m_{s}^{L} \times m_{t}^{L}+\left(\nabla g_{i} \cdot m_{t}^{L}\right)\left(m_{s}^{L} \times n_{i}\right)-\left(\nabla g_{i} \cdot m_{s}^{L}\right)\left(m_{t}^{L} \times n_{i}\right)\right\| d s d t \\
& =\left(\left\|m_{s}^{L} \times m_{t}^{L}\right\|^{2}+\left\|\left(\nabla g_{i} \cdot m_{t}^{L}\right)\left(m_{s}^{L} \times n_{i}\right)-\left(\nabla g_{i} \cdot m_{s}^{L}\right)\left(m_{t}^{L} \times n_{i}\right)\right\|^{2}\right)^{\frac{1}{2}} d s d t .
\end{aligned}
$$

Here, the derivative $\nabla g_{i}$ is taken at $m^{L}$. The last equality holds since $m_{s}^{L} \times n_{i}$ and $m_{t}^{L} \times n_{i}$ are perpendicular to $m_{s}^{L} \times m_{t}^{L}$. Furthermore,

$$
\begin{aligned}
\frac{\left\|\left(\nabla g_{i} \cdot m_{t}^{L}\right)\left(m_{s}^{L} \times n_{i}\right)-\left(\nabla g_{i} \cdot m_{s}^{L}\right)\left(m_{t}^{L} \times n_{i}\right)\right\|}{\left\|\nabla g_{i}\right\|\left\|m_{s}^{L} \times m_{t}^{L}\right\|} & \leq \frac{2\left\|\nabla g_{i}\right\|\left\|m_{s}^{L}\right\|\left\|m_{t}^{L}\right\|}{\left\|\nabla g_{i}\right\|\left\|m_{s}^{L} \times m_{t}^{L}\right\|} \\
& \leq \frac{2\left\|m_{s}^{L}\right\|\left\|m_{t}^{L}\right\|}{\left\|m_{s}^{L} \times m_{t}^{L}\right\|}=\mathscr{O}(1)
\end{aligned}
$$

because the angle $\alpha$ is bounded by (7). We now estimate (10) by making use of (11), and obtain

$$
d S=\left\|m_{s}^{L} \times m_{t}^{L}\right\|\left(1+\mathscr{O}(1)\left\|\nabla g_{i}\right\|^{2}\right)^{\frac{1}{2}} d s d t .
$$

It is well known that $g_{i}=\mathscr{O}\left(h^{2}\right)$, and hence $\nabla g_{i}=\mathscr{O}(h)$, since the triangle $T_{i}$ is a linear approximation of the surface element $\mathscr{S}_{i}$. This implies

$$
d S=\left\|m_{s}^{L} \times m_{t}^{L}\right\|\left(1+\mathscr{O}\left(h^{2}\right)\right)^{\frac{1}{2}} d s d t=\left\|m_{s}^{L} \times m_{t}^{L}\right\|\left(1+\mathscr{O}\left(h^{2}\right)\right) d s d t .
$$

Since $m^{L}$ is a linear parametrization of $P_{i}$, it follows that

$$
d S=\left(1+\mathscr{O}\left(h^{2}\right)\right) d P_{i} .
$$

From the above equations it is clear that $d S \geq d P_{i}$, and hence (9) follows.

The obvious way to proceed from here is to replace the in general irregularly shaped projection $N_{i}$ by $T_{i}$ in integral (9). Let us first note that we have only the estimate $\left|\operatorname{area}\left(N_{i}\right)-\operatorname{area}\left(T_{i}\right)\right|=\mathscr{O}\left(h^{3}\right)$, and this estimate cannot be improved to order four. Hence, a straightforward replacement of $N_{i}$ by $T_{i}$ would yield a local error $\mathscr{O}\left(h^{3}\right)$ and a global error $\mathscr{O}(h)$ which would be insufficient to prove our theorem. We will show, however, that most of the $\mathscr{O}\left(h^{3}\right)$ error terms in the approximation

$$
\int_{N_{i}} f \circ m^{i} d P_{i} \approx \int_{T_{i}} f \circ m^{i} d P_{i}
$$

cancel under the summation over $i$.

Recall that $N_{i}$ and $T_{i}=\left[v_{i, 0}, v_{i, 1}, v_{i, 2}\right]$ lie in the same plane $P_{i}$. We consider the boundary of $N_{i}$ near the edge $\left[v_{i, j-1}, v_{i, j+1}\right]$ of $T_{i}$ which is opposite to the vertex $v_{i, j}$. Note that the index $j$ is taken modulo 2 . It is not difficult to see that for sufficiently small $h$ this boundary can be described as a 
curve of the form

$$
t v_{i, j-1}+(1-t) v_{i, j+1}+\delta_{i, j}(t) n_{i, j}, \quad 0 \leq t \leq 1 .
$$

Here, $n_{i, j}$ denotes the vector in the plane $P_{i}$ with unit length which is orthogonal to the edge $\left[v_{i, j-1}, v_{i, j+1}\right]$ and points out of $T_{i}$, and $\delta_{i, j}$ denotes the distance of the curve from this edge in the direction of $n_{i, j}$. Furthermore, we introduce the orthogonal coordinate system of $P_{i}$ corresponding to (12):

$$
m_{i, j}(t, s)=t v_{i, j-1}+(1-t) v_{i, j+1}+s n_{i, j} .
$$

This is used to introduce a parametrization of $\mathscr{S}_{i}$ :

$$
\tilde{m}_{i, j}(t, s)=m_{i, j}(t, s)+g\left(m_{i, j}(t, s)\right) n_{i} .
$$

The difference between the integrals over $N_{i}$ and $T_{i}$ can now be expressed in the following formula

$$
\int_{N_{i}} f \circ m^{i} d P_{i}=\int_{T_{i}} f \circ m^{i} d P_{i}+\sum_{j} I_{i, j}
$$

where

$$
I_{i, j}:=\int_{0}^{1} \int_{0}^{\delta_{i, j}(t)} f\left(\tilde{m}_{i, j}(t, s)\right)\left\|v_{i, j-1}-v_{i, j+1}\right\| d s d t
$$

Note that

$$
\delta_{i, j}(t)=\sin \alpha_{i, j}(t)\|\underbrace{m\left(t x_{i, j-1}+(1-t) x_{i, j+1}\right)-\left(t v_{i, j-1}+(1-t) v_{i, j+1}\right)}_{=: d_{i, j}(t)}\|,
$$

where $\alpha_{i, j}(t)$ describes the angle between $d_{i, j}(t)$ and the normal $n_{i}$ of $T_{i}$. The sign of $\alpha_{i, j}(t)$ is chosen so that $d_{i, j}(t)$ points out of the prism $\left\{v+\tau n_{i}\right.$ : $\left.v \in T_{i}, \tau \in \mathbb{R}\right\}$ over $T_{i}$ for positive $\alpha_{i, j}(t)$.

The vector $d_{i, j}(t)$ is the error term of a linear interpolation, and hence $\left\|d_{i, j}(t)\right\|=\mathscr{O}\left(h^{2}\right)$. It is not possible to give an $\mathscr{O}(h)$-estimate for the angle $\alpha_{i, j}(t)$; thus a straightforward estimate of (17) only leads to $I_{i, j}=\mathscr{O}\left(h^{3}\right)$. When summing in (15) over $i$, these terms add up to $\mathscr{O}(h)$ instead of $\mathscr{O}\left(h^{2}\right)$.

To overcome this difficulty, we consider an edge $\left[v_{i, j-1}, v_{i, j+1}\right]=\left[v_{i^{\prime}, j^{\prime}-1}\right.$, $v_{i^{\prime}, j^{\prime}+1}$ of two adjacent triangles $T_{i}$ and $T_{i^{\prime}}$. Since $\alpha_{i, j}(t)$ represents the angle between $n_{i}$ and $d_{i, j}(t)$, and $\alpha_{i^{\prime}, j^{\prime}}(t)$ represents the angle between $n_{i^{\prime}}$ and $d_{i, j}(t)=d_{i^{\prime}, j^{\prime}}(t)$, it is not too difficult to see that

$$
\alpha_{i, j}(t)+\alpha_{i^{\prime}, j^{\prime}}(t)=\beta_{i, i^{\prime}},
$$

where $\beta_{i, i^{\prime}}$ is the angle between the normals of $n_{i}$ and $n_{i^{\prime}}$. Since the edges of the triangles have length $\mathscr{O}(h)$, it is well known that $\beta_{i, i^{\prime}}=\mathscr{O}(h)$. Therefore, (18) and (17) immediately imply that

$$
\delta_{i, j}(t)+\delta_{i^{\prime}, j^{\prime}}(t)=\mathscr{O}\left(h^{3}\right) .
$$


Furthermore, the following estimate can be obtained:

$$
\left\|\tilde{m}_{i, j}(t, s)-\tilde{m}_{i^{\prime}, j^{\prime}}(t,-s)\right\|=|s| \mathscr{O}(h) .
$$

The estimates (19) and (20) imply the following estimates for neighboring edges:

$$
I_{i, j}+I_{i^{\prime}, j^{\prime}}=\mathscr{O}\left(h^{4}\right) .
$$

We are now in a position to show the central estimate of this section:

$$
\int_{\mathscr{S}} f d S=\left(\sum_{i} \int_{T_{i}} f \circ m^{i} d P_{i}\right)+\mathscr{O}\left(h^{2}\right) .
$$

Proof. Given formula (9) and the notation, we have

$$
\begin{aligned}
\int_{\mathscr{S}} f d S & =\sum_{i} \int_{N_{i}} f \circ m^{i}\left(1+\mathscr{O}\left(h^{2}\right)\right) d P_{i} \\
& =\left(\sum_{i} \int_{T_{i}} f \circ m^{i} d P_{i}\right)+\mathscr{O}\left(h^{2}\right)+\sum_{i, j} I_{i, j} .
\end{aligned}
$$

It remains to estimate the last sum $\sum_{i, j} I_{i, j}$ in which the number of terms is $\mathscr{O}\left(h^{-2}\right)$. Most edges $\left[v_{i, j-1}, v_{i, j+1}\right]$ occur in pairs and because of $(21)$ only contribute an error $\mathscr{O}\left(h^{4}\right)$ per pair. The number of edges which are not common to two triangles and which hence belong to the boundary of the triangulation, is only $\mathscr{O}\left(h^{-1}\right)$. These edges contribute an error $\mathscr{O}\left(h^{3}\right)$ per term. Taking the sum now yields $\sum_{i, j} I_{i, j}=\mathscr{O}\left(h^{2}\right)$, and the proof is complete.

The conclusion of the proof of Theorem 1 now follows from standard arguments. Note that the integral

$$
\int_{T_{i}} f \circ m^{i} d P_{i}
$$

is taken over a triangle, using planar coordinates. We can hence apply the wellknown error estimates for the standard trapezoidal and midpoint rules, see, e.g., Lyness [6]:

$$
\begin{aligned}
\int_{T_{i}} f \circ m^{i} d P_{i} & =\frac{1}{3} \sum_{j=0}^{2}\left(f \circ m^{i}\right)\left(v_{i, j}\right) \operatorname{area}\left[v_{i, 0}, v_{i, 1}, v_{i, 2}\right]+\mathscr{O}\left(h^{4}\right) \\
& =\frac{1}{3} \sum_{j=0}^{2} f\left(v_{i, j}\right) \operatorname{area}\left[v_{i, 0}, v_{i, 1}, v_{i, 2}\right]+\mathscr{O}\left(h^{4}\right)
\end{aligned}
$$

for the trapezoidal rule, and

$$
\begin{aligned}
\int_{T_{i}} f \circ m^{i} d P_{i} & =\left(f \circ m^{i}\right)\left(v_{i, b}\right) \text { area }\left[v_{i, 0}, v_{i, 1}, v_{i, 2}\right]+\mathscr{O}\left(h^{4}\right) \\
& =f\left(v_{i, b}\right) \operatorname{area}\left[v_{i, 0}, v_{i, 1}, v_{i, 2}\right]+\mathscr{O}\left(h^{4}\right)
\end{aligned}
$$


for the midpoint rule. In these estimates we made use of the fact that

$$
v_{i, j}=m^{i}\left(v_{i, j}\right), \quad v_{i, b}=m^{i}\left(v_{i, b}\right)+\mathscr{O}\left(h^{2}\right)
$$

for the midpoint rule. Incorporating the estimates (23) and (24) into the central estimate (22) finishes the proof of Theorem 1.

\section{NUMERICAL EXPERIMENTS}

In this section we illustrate the behavior of the composite trapezoidal (3) and midpoint (4) rules and give numerical evidence for the following conjecture (see [3, Conjecture 5.1]):

Conjecture 1. If $\mathscr{I}(h)$ denotes the approximation (3) or (4) of the integral (1) when $\sigma$ is uniformly subdivided, then $\mathscr{I}(h)$ can be expanded in terms of $h^{2}$, i.e.,

$$
\mathscr{I}(h)=\mathscr{J}_{0}+h^{2} \mathscr{I}_{1}+\cdots+h^{2 k} \mathscr{I}_{k}+\mathscr{O}\left(h^{2 k+1}\right) .
$$

Note, that the first term in the expansion is the true value of integral (1).

In our example we integrate the function $f(x, y, z)=x^{2}+y^{2}+z^{2}$ on the surface

$$
\mathscr{S}=\left\{(x, y, z): x^{2}+y^{2}+z^{2}=1, x, y, z \geq 0\right\} .
$$

Then we have $\mathscr{J}_{0}=\frac{\pi}{2}$ for the integral. The surface $\mathscr{S}$ is parametrized in the following way: First, the standard triangle $\sigma$ is mapped affinely on the triangle $\left[a \mathbf{e}_{1}, b \mathbf{e}_{2}, c \mathbf{e}_{3}\right]$ in $\mathbf{R}^{3}$; here $a, b, c$ are positive coefficients and $\left\{\mathbf{e}_{1}, \mathbf{e}_{2}, \mathbf{e}_{3}\right\}$ denotes the standard basis of $\mathbf{R}^{3}$. This triangle is mapped via the radial projection $x \mapsto \frac{x}{\|x\|}$ onto $\mathscr{S}$. It is possible to generate very slanted and therefore ill-conditioned projections onto $\mathscr{S}$ by varying the constants $a, b, c$.

The meshsize of the standard triangle $\sigma$ is $h_{0}=1$. By subdividing $\sigma$ uniformly, we generate a sequence of meshsizes $h_{i}=2^{-i}$. For each meshsize we calculate the corresponding approximate integral $\mathscr{I}\left(h_{i}\right)$ and generate a Romberg tableau with entries $T_{i, k}$ in the $i$ th row and $k$ th column in a standard manner. Provided that the Conjecture 1 holds, we obtain the following error estimate (see $[7, \S 3.4])$ :

$$
T_{i, k}-\mathscr{J}_{0}=(-1)^{k} h_{i-k}^{2} h_{i-k+1}^{2} \cdots h_{i}^{2}\left(1+\mathscr{O}\left(h_{i-1-k}\right)\right) .
$$

In order to verify this last estimate, we calculate the logarithmic ratio

$$
\Delta_{i, k}=\log _{4} \frac{T_{i-1, k}-\mathscr{I}_{0}}{T_{i, k}-\mathscr{I}_{0}}
$$

and compare the numerical result with the assymptotic behavior

$$
\Delta_{i, k}=k+1+\mathscr{O}\left(h_{i-k-1}\right) .
$$

The following Romberg tableaus display the results for two different sets of coefficients $a, b, c$ and for both composite rules (3) and (4). 
Trapezoidal Rule: $a=1, b=1, c=1$

\begin{tabular}{|c|c|c|c|c|c|c|c|c|c|c|}
\hline \multicolumn{6}{|c|}{ Absolute Errors } & \multicolumn{5}{|c|}{ Logarithmic Ratio $\Delta_{i, k}$} \\
\hline$\overline{2.6 \mathrm{e}-01}$ & & & & & & & & & & \\
\hline $7.7 e-02$ & $1.3 \mathrm{e}-02$ & & & & & 0.897 & & & & \\
\hline $2.0 \mathrm{e}-02$ & $1.2 \mathrm{e}-03$ & $4.3 e-04$ & & & & 0.965 & 1.721 & & & \\
\hline $5.1 \mathrm{e}-03$ & $8.5 \mathrm{e}-05$ & $6.7 \mathrm{e}-06$ & $5.6 \mathrm{e}-08$ & & & 0.991 & 1.945 & 3.006 & & \\
\hline $1.3 \mathrm{e}-03$ & $5.4 \mathrm{e}-06$ & $1.1 \mathrm{e}-07$ & $3.1 \mathrm{e}-09$ & $3.4 \mathrm{e}-09$ & & 0.998 & 1.986 & 2.979 & 2.079 & \\
\hline $3.2 \mathrm{e}-04$ & $3.4 \mathrm{e}-07$ & $1.7 \mathrm{e}-09$ & $4.1 \mathrm{e}-11$ & $2.9 \mathrm{e}-11$ & $2.6 \mathrm{e}-11$ & 0.999 & 1.997 & 2.983 & 3.128 & 3.433 \\
\hline
\end{tabular}

Midpoint Rule: $a=1, b=1, c=1$

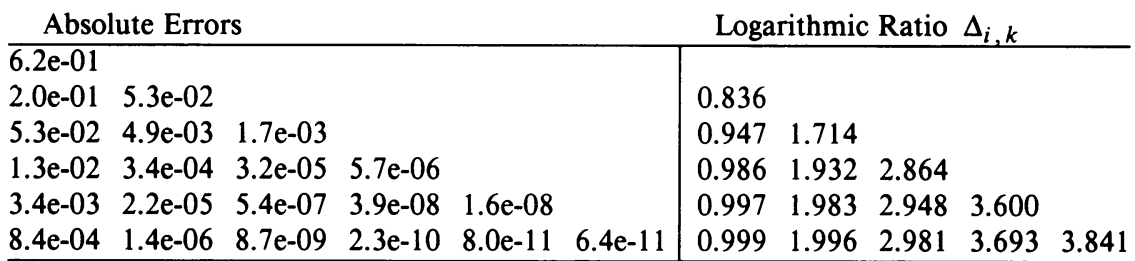

Trapezoidal Rule: $a=0.5, b=1, c=2$

\begin{tabular}{|c|c|c|c|c|c|c|c|c|c|c|}
\hline Absol & lute Erro & & & & & Loga & rithmic & Ratio & $\Delta_{i, k}$ & \\
\hline $3.9 \mathrm{e}-01$ & & & & & & & & & & \\
\hline $1.5 \mathrm{e}-01$ & $7.4 \mathrm{e}-02$ & & & & & 0.678 & & & & \\
\hline $4.3 \mathrm{e}-02$ & $6.9 \mathrm{e}-03$ & $2.4 \mathrm{e}-03$ & & & & 0.909 & 1.711 & & & \\
\hline $1.1 \mathrm{e}-02$ & $4.7 \mathrm{e}-04$ & $4.2 \mathrm{e}-05$ & $4.7 \mathrm{e}-06$ & & & 0.977 & 1.936 & 2.916 & & \\
\hline $2.8 \mathrm{e}-03$ & $3.3 \mathrm{e}-05$ & $3.6 \mathrm{e}-06$ & $3.0 \mathrm{e}-06$ & $3.0 \mathrm{e}-06$ & & 0.994 & 1.921 & 1.776 & 0.326 & \\
\hline $7.1 \mathrm{e}-04$ & $2.1 \mathrm{e}-06$ & $4.7 \mathrm{e}-08$ & $9.8 \mathrm{e}-09$ & $2.1 \mathrm{e}-08$ & $2.5 \mathrm{e}-08$ & 0.998 & 1.985 & 3.135 & 4.127 & 3.556 \\
\hline
\end{tabular}

Midpoint Rule: $a=0.5, b=1, c=2$

\begin{tabular}{|c|c|c|c|c|c|c|c|c|c|c|}
\hline Abso & lute Erro & & & & & $\log$ & rithmic & Ratio & $\Delta_{i, k}$ & \\
\hline $8.6 \mathrm{e}-01$ & & & & & & & & & & \\
\hline $3.8 \mathrm{e}-01$ & $2.3 e-01$ & & & & & 0.577 & & & & \\
\hline $1.2 \mathrm{e}-01$ & $2.8 \mathrm{e}-02$ & $1.4 \mathrm{e}-02$ & & & & 0.860 & 1.527 & & & \\
\hline $3.1 \mathrm{e}-02$ & $2.1 \mathrm{e}-03$ & $4.1 \mathrm{e}-04$ & $1.90 \mathrm{e}-04$ & & & 0.962 & 1.854 & 2.550 & & \\
\hline $7.8 \mathrm{e}-03$ & $1.5 e-04$ & $1.5 \mathrm{e}-05$ & $8.4 \mathrm{e}-06$ & $7.7 \mathrm{e}-06$ & & 0.990 & 1.928 & 2.404 & 2.265 & \\
\hline $2.0 \mathrm{e}-03$ & $9.3 e-06$ & $2.2 \mathrm{e}-07$ & $9.9 \mathrm{e}-09$ & $4.3 \mathrm{e}-08$ & $5.0 \mathrm{e}-08$ & 0.997 & 1.984 & 3.031 & 4.866 & 3.742 \\
\hline
\end{tabular}

These tableaus confirm our Conjecture 1, given the finite-precision arithmetic of the computer used and the $\mathscr{O}\left(h_{i-k-1}\right)$ error of the asymptotic behavior of (25).

\section{BIBLIOGRAPHY}

1. K. E. Atkinson, A survey of boundary integral equation methods for the numerical solution of Laplace's equation in three dimensions, Numerical Solution of Integral Equations (New York) (M. Goldberg, ed.), Plenum Press, 1990, pp. 1-34.

2. _ Two-grid iteration method for linear integral equations of the second kind on piecewise smooth surfaces in $\mathbf{R}^{3}$, Report 14, Univ. of Iowa, submitted to SIAM J. Numer. Anal., 1991. 
3. K. Georg, Approximation of integrals for boundary element methods, SIAM J. Sci. Statist. Comput. 12 (1991), 443-453.

4. K. Georg and R. Widmann, Adaptive quadratures over surfaces, Colorado State University, 1993, preprint.

5. W. Hackbusch, Integralgleichungen. Theorie und Numerik, B. G. Teubner, Stuttgart, Germany, 1989.

6. J. N. Lyness, Quadrature over a simplex: Part 2. A representation for the error functional, SIAM J. Numer. Anal. 15 (1978), 870-887.

7. J. Stoer and R. Bulirsch, Introduction to numerical analysis, Springer-Verlag, Berlin, Heidelberg, New York, 1980.

Department of Mathematics, Colorado State University, Ft. Collins, Colorado 80523

E-mail address: georg@math.colostate.edu 